\title{
A Kinetic Model of the Monocarboxylate Transporter MCT1 and its Interaction with Carbonic Anhydrase II
}

\author{
Joachim Almquist ${ }^{1 *}$, Patrick Lang ${ }^{2}$, Dieter Prätzel-Wolters ${ }^{3}$, Joachim W. Deitmer ${ }^{4}$, Mats Jirstrand ${ }^{1}$ and Holger M. Becker ${ }^{4}$ \\ ${ }^{1}$ Fraunhofer-Chalmers Centre, Chalmers Science Park, Göteborg, Sweden \\ ${ }^{2}$ Abteilung "Systemanalyse, Prognose und Regelung", Fraunhofer Institut für Techno- und Wirtschafts-mathematik (ITWM), Fraunhofer-Platz 1, Kaiserslautern, Germany \\ ${ }^{3}$ Technomathematik, University of Kaiserslautern, Erwin-Schroedingerstr, Kaiserslautern, Germany \\ ${ }^{4}$ Division of General Zoology, Department Biology, University of Kaiserslautern, POB 30 49, D Kaiserslautern, Germany
}

\begin{abstract}
The enzyme carbonic anhydrase isoform II (CAII), catalyzing the hydration and dehydration of $\mathrm{CO}_{2}$, enhances transport activity of the monocarboxylate transporter isoform I (MCT1, SLC16A1) expressed in Xenopus oocytes by a mechanism that does not require CAll catalytic activity. In the present study, we have investigated the mechanism of the CAll induced increase in transport activity by using electrophysiological techniques and mathematical modeling of the MCT1 transport cycle. The model consists of six states arranged in cyclic fashion and features an ordered, mirrorsymmetric, binding mechanism, where binding and unbinding of the proton to the transport protein is considered to be the rate limiting step under physiological conditions. An explicit rate expression for the substrate flux is derived using model reduction techniques. By treating the pools of intra-and extracellular MCT1 substrates as dynamic states, the time dependent kinetics are obtained by integration, using the derived expression for the substrate flux. The simulations were compared with experimental data obtained from MCT1-expressing oocytes injected with different amounts of CAll. The model suggests that CAll increases the effective rate constants of the proton reactions, possibly by working as a proton antenna.
\end{abstract}

Keywords: CAII; Mathematical modeling; Model reduction; Electrophysiology; pH-sensitive microelectrodes; Proton antenna

\section{Introduction}

Transport of acid/base equivalents across cell membranes plays a pivotal role both in intra- and extracellular $\mathrm{pH}$ regulation and for the import and export of metabolites, as many $\mathrm{Na}^{+}$-dependent and $\mathrm{Na}^{+}$independent substrate transporters use $\mathrm{H}^{+}, \mathrm{OH}^{-}$or $\mathrm{HCO}_{3}^{-}$as co- or counter-substrate. One family of these $\mathrm{H}^{+}$-substrate cotransporters are the monocarboxylate transporters (MCT, SLC16) which comprise 14 isoforms. MCTs, that carry the energetic compounds lactate, pyruvate, and keton bodies together with protons in an electroneutral 1 proton : 1 monocarboxylate stoichiometry, are expressed in most tissues, especially those with large energy consumption like muscle and brain (Halestrap and Meredith, 2004; Bergersen, 2007). In the brain, MCT1 is located mainly in glial cells, where it facilitates the export of lactate, which is then taken up by neurons via the high affinity MCT2; thereby the two isoforms are believed to shuttle lactate from glial cells to neurons, a mechanism that seems to be crucial for brain energy metabolism (Schurr et al., 1988; Magistretti et al., 1999; Pellerin et al., 1998; Bouzier-Sore et al., 2003; Debernardi et al., 2003). In muscle, the predominant isoforms are MCT1, MCT3, and MCT4. MCT1 is highly expressed in oxidative type I fibers, while the density of MCT3 and MCT4 is independent of the fiber type (Hashimoto et al., 2005; Wilson et al., 1998; Juel, 2001). MCT3 and MCT4 seem to be responsible for the removal of lactate from glycolytic muscle cells, while MCT1 mediates lactate-import into oxidative fibers. Thereby the different isoforms facilitate lactate shuttling between glycolytic and oxidative muscle fibers (Brooks, 1998). The transport of monocarboxylates between different cell types in the muscle and between muscle and blood has been found to have a significant impact on regulation of muscular pH (Thomas et al., 2007; Bangsbo et al., 1997; Juel et al., 2001).

The isoform MCT1, used in the present study, has extensively been studied in different systems. MCT1 has a classical 12 transmembrane-helix structure, with both the $\mathrm{N}$ - and C-terminus being located intracellularly (Poole et al., 1996). Expression of MCT1 in Xenopus oocytes revealed a $\mathrm{K}_{\mathrm{m}}$ value for lactate of $3.5 \mu \mathrm{M}$ and a strong dependency of lactate transport from the extracellular $\mathrm{H}^{+}$ concentration (Bröer et al., 1998). Lactate transport via the MCTs has been described by different kinetic models. By measuring influx of ${ }^{14} \mathrm{C}$-labeled lactate into red blood cells, a kinetic model was proposed that describes the lactate transport as a symport system with ordered binding of lactate and $\mathrm{H}^{+}$, in the sense that the proton binds first to the carrier, creating the binding site for the negatively charged lactate, followed by binding of lactate (de Bruijne et al., 1983). After translocation of lactate and $\mathrm{H}^{+}$across the membrane, lactate is released first from the transporter followed by the proton. As the rates of monocarboxylate exchange are substantially faster than those of net transport, the return of the free carrier across the membrane was considered as the rate-limiting step for net lactic acid flux (Juel and Halestrap, 1999). Investigation of point mutations within critical residues of the MCT1 revealed that the transmembrane helix 8 and the intracellular loop between transmembrane helices 10 and 11

*Corresponding author: Joachim Almquist, Fraunhofer-Chalmers Centre, Chalmers Science Park, SE-412 88 Göteborg, Sweden, E-mail: joachim.almquist@ fcc.chalmers.se

Received October 26, 2010; Accepted November 25, 2010; Published November 27,2010

Citation: Almquist J, Lang P, Prätzel-Wolters D, Deitmer JW, Jirstrand M, et al. (2010) A Kinetic Model of the Monocarboxylate Transporter MCT1 and its Interaction with Carbonic Anhydrase II. J Comput Sci Syst Biol 3: 107-116. doi:10.4172/jcsb.1000066

Copyright: @ 2010 Almquist $\mathrm{J}$, et al. This is an open-access article distributed unde the terms of the Creative Commons Attribution License,which permits unrestricted use, distribution, and reproduction in any medium, provided the original author and source are credited. 
are crucial for binding of protons and substrate transport across the protein (Galic et al., 2003).

MCT1 has been shown to cooperate with the sodium-bicarbonate cotransporter NBCe1 (Becker et al., 2004), and with carbonic anhydrase isoform II (CAII) (Becker et al., 2005). Remarkably, the increase of MCT1 transport activity by CAII was independent of the catalytic activity which catalyzes the reaction between $\mathrm{CO}_{2}, \mathrm{H}^{+}$and $\mathrm{HCO}_{3}^{-}$(Becker et al., 2005). CAII seems to bind to, and enhance the activity of, various acid/base transporters like the sodium-bicarbonate cotransporter NBCe1 (Becker and Deitmer, 2007; Pushkin et al., 2004), the chloride/bicarbonate exchanger AE1 (Vince and Reithmeier, 1998) and the sodium/hydrogen exchanger NHE1 (Li et al., 2002). In muscle cells, co-expression of CAII and acid/base transporting proteins like NBC, NHE1 and also the MCTs have been found to be pivotal in acid/ base homeostasis (Juel et al., 2003). In addition, it has been shown that extracellular carbonic anhydrase activity facilitates lactic acid transport in rat skeletal muscle fibers (Wetzel et al., 2001). In the brain CAII is highly expressed in astrocytes where it plays a supportive role in $\mathrm{pH}$ regulation (Deitmer and Rose, 1996) and in lactate shuttling between astrocytes and neurons (Svichar and Chesler, 2003).

In the present study, we present a mathematical model based on rate equations and model reduction that describes $\mathrm{H}^{+}$-lactate cotransport via the MCT1 and tries to give an explanation for the mechanism by which CAII increases MCT1 activity. $\mathrm{H}^{+}$-lactate cotransport via the MCT1 can be described as a multi-step process including substrate binding, translocation of bound substrate over the membrane, and substrate release (Fall et al., 2002). This chain of events can be described by a number of states, representing a protein or protein-substrate complex with its binding sites or bound substrates facing either the intracellular or extracellular side of the membrane. At any given time, a single protein belongs to precisely one of these states, but the total population of MCT1 proteins are divided into sub-populations distributed over all the different states. If the number of proteins residing in every state is sufficiently large, the rate of change of each state can be described by a deterministic, ordinary differential equation (ODE) based on mass action kinetics (Klipp et al., 2005; Szallasi et al., 2006). The set of all rate equations, then, constitutes the model of the membrane transport process. Based on the transport measurement techniques used in this study, it is not possible to determine the values of all rate constants appearing in the type of ODE model described above. It is therefore desirable to reduce unnecessary complexity of the model. Model reduction techniques aim at simplifying models to reach an appropriate level of detail for experimental validation (Fall et al., 2002; Klipp et al., 2005). Two common methods of reducing the complexity of a model by reducing the number of ODEs, both based on timescale separation, are the quasi-equilibrium approximation and the quasisteady-state approximation (Bower and Bolouri, 2001; Klipp et al., 2005). Using these methods in combination with electrophysiological experiments, we arrive at an explicit, analytic expression for the transport rate of MCT1. Furthermore, the model is extended to also incorporate the effect of CAII and the results of simulations are compared to measurement data.

\section{Materials and Methods}

\section{Constructs, oocytes and injection of cRNA and carbonic anhydrase}

Rat MCT1 cDNA (MCT1) cloned in oocyte expression vector pGEM-He-Juel, which contains the 5' and the 3' untranscribed regions of the Xenopus $\beta$-globulin flanking the multiple cloning site, was kindly provided by Dr. Stefan Bröer, Canberra (Bröer et al., 1997). Plasmid DNA was linearised with NotI and transcribed in vitro with T7 RNA-Polymerase in the presence of the cap analogon $\mathrm{m} 7 \mathrm{G}\left(5^{\prime}\right) \mathrm{ppp}\left(5^{\prime}\right) \mathrm{G}$ (mMessage mMachine, Ambion Inc., USA) to produce a capped RNA transcript. The cRNA was purified with the Qiagen RNeasy MinElute Cleanup Kit (Qiagen $\mathrm{GmbH}$, Hilden, Germany) and stored at $-80^{\circ} \mathrm{C}$ in DEPC$\mathrm{H}_{2} \mathrm{O}$. Xenopus laevis females were purchased from Xenopus Express Inc. (Vernas-sal, France). Oocytes were isolated and singularized by collagenase (Collagenase A, Roche, Mannheim, Germany) treatment in $\mathrm{Ca}^{2+}$-free oocyte saline at 28 for $2 \mathrm{~h}$. The singularized oocytes were left overnight in an incubator at 18 in $\mathrm{Ca}^{2+}$-containing oocyte saline ( $\mathrm{pH} \mathrm{7.8)} \mathrm{to} \mathrm{recover.} \mathrm{The} \mathrm{oocyte} \mathrm{saline,} \mathrm{also} \mathrm{used} \mathrm{for} \mathrm{the}$ electrophysiological experiments, had the following composition (in $\mathrm{mM}$ ): $\mathrm{NaCl}, 82.5 ; \mathrm{KCl}, 2.5 ; \mathrm{CaCl}_{2}, 1 ; \mathrm{MgCl}_{2}, 1, \mathrm{Na}_{2} \mathrm{HPO}_{4} ; 1$, HEPES, 5, titrated with $\mathrm{NaOH}$ to the desired $\mathrm{pH}$. In the bicarbonate-containing saline, $\mathrm{NaCl}$ was replaced by an equivalent amount of $\mathrm{NaHCO}_{3}$ and the solution was aerated with $5 \% \mathrm{CO}_{2}$. For lactate containing solutions $\mathrm{NaCl}$ was replaced equimolar by Na-L-Lactate. Oocytes of the stages $\mathrm{V}$ and VI were selected and injected with $7 \mathrm{ng}$ of MCT1-cRNA dissolved in DEPC- $\mathrm{H}_{2} \mathrm{O}$ using glass micropipettes and a microinjection device (Nanoliter 2000, World Precision Instruments, Berlin, Germany). Control oocytes were injected with an equivalent volume of DEPC$\mathrm{H}_{2} \mathrm{O} .50$ or $200 \mathrm{ng}$ of CAII, isolated from bovine erythro-cytes (C3934, Sigma-Aldrich, Taufkirchen, Germany), dissolved in $25 \mathrm{nl}$ DEPC- $\mathrm{H}_{2} \mathrm{O}$, were injected 20-24 h before electrophysiological measurement. Control oocytes were injected with $25 \mathrm{nl}$ DEPC- $\mathrm{H}_{2} \mathrm{O}$.

\section{Intracellular pH measurements}

For measurement of intracellular $\mathrm{pH}\left(\mathrm{pH}_{\mathrm{i}}\right)$ and membrane potential double-barreled microelectrodes were used; the manufacture and application have been described in detail previously (Deitmer, 1991). Briefly, for double-barreled microelectrodes, two borosilicate glass capillaries of 1.0 and $1.5 \mathrm{~mm}$ in diameter were twisted together and pulled to a micropipette. The ion-selective barrel was silanized with a drop of $5 \%$ tri-N-butylchlorsilane in $99.9 \%$ pure carbon tetrachloride, backfilled into the tip. The micropipette was baked for $4.5 \mathrm{~min}$ at $450^{\circ} \mathrm{C}$ on a hot plate. $\mathrm{H}^{+}$-sensitive cocktail (Fluka 95291, Fluka, Buchs, Switzerland) was backfilled into the tip of the silanized ion-selective barrel and filled up with $0.1 \mathrm{M} \mathrm{Na}$-citrate, $\mathrm{pH}$ 6.0. The reference barrel was filled with $3 \mathrm{M} \mathrm{KCl}$. To increase the opening of the electrode-tip, it was bevelled with a jet stream of aluminium powder suspended in $\mathrm{H}_{2} \mathrm{O}$. The central and the reference barrel of the electrodes were connected by chlorided silver wires to the head stages of an electrometer amplifier. Calibration of the electrodes was carried out in oocyte salines by changing the $\mathrm{pH}$ by 0.6 units. Electrodes were accepted for use in the experiments, when their response exceeded $50 \mathrm{mV}$ per unit change in $\mathrm{pH}$; on average, they responded with $54 \mathrm{mV}$ for unit change in pH. As described previously (Bröer et al., 1998), optimal pH changes were detected when the electrode was located near the inner surface of the plasma membrane. This was achieved by carefully rotating the oocyte with the impaled electrode. All experiments were carried out at room temperature.

\section{Calculation of $\left[\mathrm{H}^{+}\right]_{\mathrm{i}}$}

The measurements of $\mathrm{pH}_{\mathrm{i}}$ were stored digitally using in house PC software based on the program LabView (National Instruments Germany GmbH, München, Germany) and were routinely converted into intracellular $\mathrm{H}^{+}$concentration, $\left[\mathrm{H}^{+}\right]_{i}$. Thus, changes in the $\left[\mathrm{H}^{+}\right]_{i}$ are recorded, which take into account the different $\mathrm{pH}$ baseline, as e.g. measured in HEPES-and $\mathrm{CO}_{2} / \mathrm{HCO}_{3}^{-}$buffered salines (see also (Becker 
et al., 2004)). The rate of change of the measured $\left[\mathrm{H}^{+}\right]_{\mathrm{i}}$ was analyzed by determining the slope of a linear regression fit using the spread sheet program OriginPro 7 (OriginLab Corporation, Northampten, USA).

\section{Voltage-Clamp recording}

A borosilicate glass capillary, $1.5 \mathrm{~mm}$ in diameter, was pulled to a micropipette and back-filled with $3 \mathrm{M} \mathrm{KCl}$. The resistance of the electrodes measured in oocyte saline was around $1 \mathrm{M}$. For voltageclamp, the electrode was connected to the head-stage of an Axoclamp 2B amplifier (Axon Instruments, USA). The experimental bath was grounded with a chlorided silver wire coated by agar dissolved in oocyte saline. Oocytes were clamped to a holding potential of -40 $\mathrm{mV}$.

\section{Statistical calculations}

Statistical values are presented as means and standard errors of the means (S.E.M.). For calculation of significance in differences, Students $t$-test or, if possible, a paired $t$-test was used.

\section{Mathematics and modeling}

By distinguishing between fast and slow reactions in an ODE model, two or more states interconnected by fast reactions can be treated by the quasi-equilibrium approximation on the timescale of the slower reactions (Bower and Bolouri, 2001; Klipp et al., 2005). Mathematically, this can be formulated as an algebraic constraint for each fast reaction. For a cyclic chain of $n$ states, it is at most possible to find $n-1$ independent constraints from fast reactions. Depending on the number of slow reactions it could be fewer. All states in the system, even those linked to "slow" reactions, reach their steady state a lot faster than relevant changes in substrate concentrations. This is a consequence of the fact that the amount of substrate outnumbers the amount of MCT1 expressed in the oocyte cell membrane by far. On the timescale of relevant changes in substrate concentrations, it is therefore reasonable to consider the subsets of quasi-equilibrium interconnected states, connected by the slower reactions, to be in quasi-steady-state. This will provide additional algebraic constraints. By determining as many linearly independent constraints as there are numbers of states, it was possible to solve for a unique solution of occupancy level of all states. Once this task was achieved, the dynamic, multi-state description of the system was reduced to an explicit, analytic rate expression for the trans-membrane transport of substrates, being a function of slow rate constants, equilibrium constants, and substrate concentrations. This is a much more general way of characterizing the transport process than merely stating a $\mathrm{V}_{\max }$ and an apparent $\mathrm{K}_{\mathrm{m}}$. The transport rate expression gained from the model reduction was then used to describe the rate of change of intracellular substrate concentrations, induced by changes in the substrate gradient. The complexity of the rate expression required that this had to be done by numeric integration.

In addition to the changes in substrate concentrations related to membrane transport, changes in concentrations due to other chemical reactions in the cytosol bulk had to be considered. Lactate has a rather low $\mathrm{pK}_{\mathrm{a}}$ value of 3.86. Since the experiments are carried out at extracellular $\mathrm{pH}\left(\mathrm{pH}_{\mathrm{o}}\right) 5$ to 8 , the only species considered is the dissociated acid form. Protons on the other hand, received special treatment because of their strong linkage with buffers. The intrinsic buffer system of the oocyte was modeled as a single species with a single $\mathrm{pK}_{\mathrm{a}}, \mathrm{B}^{-}+\mathrm{H}^{+} \rightleftharpoons \mathrm{HB}$. Buffering was assumed to be instantaneous, and the $\mathrm{pK}_{\mathrm{a}}$ was set to a value around 5 to keep the buffer from saturating. The extracellular space is not a dynamic compartment in the same sense as the intracellular space. Perfusion of the bath solution maintains a constant, controlled environment around the oocyte. For this reason, the extracellular space was used as a signal input compartment reflecting the experiment protocol. To make the model more realistic, switching of the bath solution was modeled as a first order system with specific time constant. This time constant was measured at the same time as the electrodes were calibrated.

The Systems Biology Toolbox for MATLAB (Schmidt, 2005) was used to create the cell model and to perform the numerical integrations, together with the extension package SBaddon (Schmidt, 2006). The toolbox features several modeling and simulation tools and a special representation of the experimental protocols. The $\Delta \mathrm{H}^{+} / \Delta \mathrm{t}$ following input signals was extracted from simulated time series, and then compared to real measurements. The analytic solution of systems of algebraic equations were obtained with Mathematica (Wolfram Research, Inc., Champaign, USA).

\section{Results}

The aim of this study was to determine the mechanism of MCT1, and how it interacts with the enzyme CAII, based on a combination of electrophysiological techniques and mathematical modeling. The transport process of MCT1 was modeled as a number of interconnected states, representing a network of discrete proteinsubstrate configurations. Figure 1 shows a hypothetical model of this type for the MCT1 transport. Each of the six MCT1-states, indicated by an $M$ with an index, represents the protein or a protein-substrate complex. An efflux transport cycle starts with an intracellular proton binding to the MCT1, $M_{1}$, causing a transition to $M_{2}$. After binding of lactate the protein-substrate complex moves from $M_{3}$ to $M_{4}$, making the substrates face the extracellular side. The substrates then dissociate from the protein in a reversed order through $M_{5}$ and $M_{6}$, before the translocation of the binding-sites to $M_{1}$, completing the cycle. Since all reactions are reversible, the exact behavior of an individual MCT1 protein cannot be calculated. Therefore the model describes the state transitions by a large population of proteins.

The rate of change of each state in Figure 1 is described by an ordinary differential equation

$$
\begin{aligned}
& \frac{d M_{1}}{d t}=k_{21} M_{2}+k_{61} M_{6}-k_{12}\left[H_{i n}^{+}\right] M_{1}-k_{16} M_{1} \\
& \frac{d M_{2}}{d t}=k_{12}\left[H_{i n}^{+}\right] M_{1}+k_{32} M_{3}-k_{21} M_{2}-k_{23}\left[L a c_{i n}^{-}\right] M_{2} \\
& \frac{d M_{3}}{d t}=k_{23}\left[L a c_{i n}^{-}\right] M_{2}+k_{43} M_{4}-k_{32} M_{3}-k_{34} M_{3} \\
& \frac{d M_{4}}{d t}=k_{34} M_{3}+k_{54}\left[L a c_{e x}^{-}\right] M_{5}-k_{43} M_{4}-k_{45} M_{4} \\
& \frac{d M_{5}}{d t}=k_{45} M_{4}+k_{65}\left[H_{e x}^{+}\right] M_{6}-k_{54}\left[L a c_{e x}^{-}\right] M_{5}-k_{56} M_{5}(5) \\
& \frac{d M_{6}}{d t}=k_{16} M_{1}+k_{56} M_{5}-k_{61} M_{6}-k_{65}\left[H_{e x}^{+}\right] M_{6}
\end{aligned}
$$

The dynamics of this system will be influenced by the relative speed of the reactions. By assuming that one or several of the reactions are slower than the others, the remaining reactions can, by the quasiequilibrium approximation, be considered to always be in equilibrium. To illustrate how the model reduction is done in practice, we assume that the reactions between $M_{1}$ and $M_{2}$ and between $M_{5}$ and $M_{6}$, are slower than the other reactions. This assumption yields four quasi- 


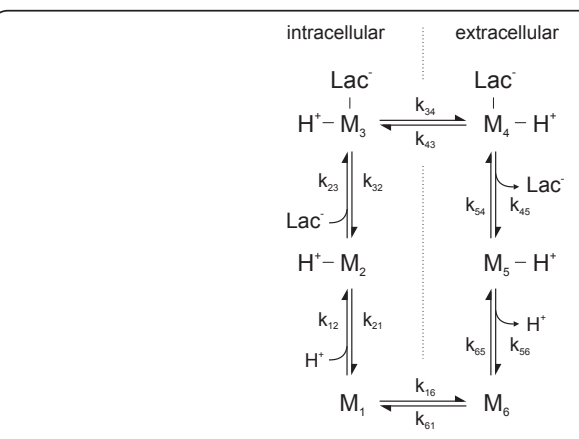

Figure 1: Kinetic model of $\mathrm{H}^{+}$-lactate co-transport via the MCT1. $M_{1}$ to $M_{6}$ represent the six states of MCT1 during substrate transport, $k_{x y}$ are rate constants for the reactions between two transporter states.

equilibrium constraints for the system

$$
\begin{aligned}
& k_{16} M_{1}=K_{61} M_{6} \\
& k_{34} M_{3}=K_{43} M_{4} \\
& k_{23}\left[L a c_{i n}^{-}\right] M_{2}=k_{32} M_{3} \\
& k_{54}\left[\operatorname{Lac}_{e x}^{-}\right] M_{5}=k_{45} M_{4}
\end{aligned}
$$

where we introduce the notation $K_{i}=k_{23} / k_{32}, K_{o}=k_{54} / k_{45}, K_{l}=k_{34}$ $/ k_{43}$, and $K_{e}=k_{61} / k_{16}$ for later convenience. Also assuming that the total number of proteins is constant over time, i.e.,

$$
\sum_{i} M_{i}=M_{t o t}
$$

and that the subsets of quasi-equilibrium states are in quasi-steadystate,

$$
k_{12}\left[H_{i n}^{+}\right] M_{1}-k_{21} M_{2}=k_{56} M_{5}-k_{65}\left[H_{e x}^{+}\right] M_{6}
$$

gives a total of six independent algebraic constraints. Solving the system of linear equations Eq. 7-12 yields a distribution $M^{s}$ of the MCT1s over the six states as a function of the intra and extracellular concentrations of protons and lactate. The rate of net flux $T$ over the membrane is then given by

$$
T=k_{12}\left[H_{i n}^{+}\right] M_{1}^{s}-k_{21} M_{2}^{s}
$$

or equivalently

$$
T=k_{56} M_{5}^{s}-k_{65}\left[H_{e x}^{+}\right] M_{6}^{s}
$$

These two expressions are equivalent, because of the constraint in Eq. 12. With the solution of Eq. 7-12 inserted into Eq. 13 or Eq. 14, the expression for the net transport becomes

$$
T=M_{t o t} \frac{k_{12} k_{56} K_{i} K_{l}\left[H_{i n}^{+}\right]\left[L a c_{i n}^{-}\right]-k_{21} k_{65} K_{o} K_{e}\left[H_{e x}^{+}\right]\left[L a c_{e x}^{-}\right]}{A\left[H_{i n}^{+}\right]+B\left[H_{e x}^{+}\right]+C\left[L a c_{i n}^{-}\right]+D\left[L a c_{e x}^{-}\right]}
$$

where $A, B, C$ and $D$ are given by

$$
\begin{aligned}
& A=k_{12}\left(K_{i} K_{I}\left[L a c_{i n}^{-}\right]+K_{o}\left[L a c_{e x}^{-}\right]+K_{i} K_{o}\left(K_{I}+1\right)\left[L a c_{i n}^{-}\right]\left[L a c_{e x}^{-}\right]\right) \\
& B=k_{65}\left(K_{i} K_{e} K_{I}\left[L a c_{i n}^{-}\right]+K_{o} K_{e}\left[L a c_{e x}^{-}\right]+K_{i} K_{o} K_{e}\left(K_{I}+1\right)\left[L a c_{i n}^{-}\right]\left[\operatorname{Lac}_{e x}^{-}\right]\right)
\end{aligned}
$$

$$
C=k_{56} K_{i} K_{l}\left(K_{\mathrm{e}}+1\right)
$$

$$
D=k_{21} K_{o}\left(K_{e}+1\right)
$$

As a consequence of the second law of thermodynamics, the rate and equilibrium constants in Eq. 15 must obey the following equation.

$$
k_{12} k_{56} K_{i} K_{l}=k_{21} k_{65} K_{o} K_{e}
$$

Other combinations of substrate binding-order and assumptions about slow reactions lead, in a similar way, to rate expressions of different functional form. To find the best description of the system, the set of these combinations must be explored. As possible slow, or rate-limiting, steps, we consider the binding and release of protons, the binding and release of lactate, the translocation of the loaded carrier, and the translocation of the empty carrier. As possible binding orders, we consider only the two types with mirror- symmetric binding (first in, last out). This gives eight candidate models. The omission of glide-symmetry and none-ordered binding (random binding) is discussed later.

In order to discriminate between the different models we measured the rate of change in intracellular $\mathrm{H}^{+}$concentration, as an indicator for transport activity, in MCT1 expressing oocytes during efflux of $\mathrm{H}^{+}$and lactate. The efflux experiment was chosen in order to control the composition of the trans-side solution via the extracellular bath. By using a bath solution without lactate, or with lactate but at a very high $\mathrm{pH}$, a situation with practically only one of the two substrates present was created. In the models, this corresponds to setting either $\left[\mathrm{Lac}_{e x}^{-}\right]$or $\left[\mathrm{H}_{e x}^{+}\right]$to zero. A thorough analysis of the considered models reveals that some of them show an efflux transport rate which is dependent on the single extracellular substrate still remaining. This means that in some model configurations, a single substrate on the trans-side may act as an inhibitor of transport. Table 1 shows possible single substrate inhibitions for the different model configurations. All model configurations are sensitive to one of the substrates, except for two configurations that predict inhibition features for both protons and lactate. This suggests that certain model configurations can be ruled out on the basis of single substrate inhibition experiments.

To discriminate between the different models, we measured efflux of $\mathrm{H}^{+}$in a MCT1 expressing oocyte under different conditions as shown in Figure 2. The cell was loaded with $3 \mathrm{mM}$ lactate at an $\mathrm{pH}_{\mathrm{o}}$ of 7.0. After equilibrium was reached, the extracellular solution was changed either to $0 \mathrm{mM}$ lactate, $\mathrm{pH}_{0} 9.0$ (Figure 2A), $60 \mathrm{mM}$ lactate, $\mathrm{pH}_{\mathrm{o}} 9.0$ (Figure 2B), or $0 \mathrm{mM}$ lactate $\mathrm{pH}_{0} 6.0$ (Figure 2C). The experiment was repeated three times with the same results. Loading of lactate resulted in an intracellular acidification that reached a plateau at a $\mathrm{pH}_{\mathrm{i}}$ of 7.3 , which equals an $\left[\mathrm{H}^{+}\right]_{\mathrm{i}}$ of $50 \mathrm{nM}$. From this value an intracellular lactate concentration of $6 \mathrm{mM}$ can be calculated from the Donnan equation. Simultaneously removing both substrates by changing extracellular solution to $0 \mathrm{mM}$ lactate,

\begin{tabular}{|l|l|l|l|}
\hline $\begin{array}{l}\text { Assumed properties of } \\
\text { transport kinetics }\end{array}$ & Rate-limiting step & $\begin{array}{l}\text { Predicted effect } \\
\text { on transport }\end{array}$ & Inhibition by $\mathrm{H}^{+}$ \\
\hline First in, last out & $\begin{array}{l}\text { Inhibition } \\
\text { by Lac }\end{array}$ \\
\hline Proton $\left(\mathrm{H}^{+}\right)$ & binding/release of $\mathrm{H}^{+}$ & Yes & Yes \\
\hline & binding/release of Lac & Yes & No \\
\hline & $\begin{array}{l}\text { translocation of empty } \\
\text { carrier }\end{array}$ & Yes & No \\
\hline & $\begin{array}{l}\text { translocation of loaded } \\
\text { carrier }\end{array}$ & Yes & No \\
\hline Lactate (Lac-) & binding/release of $\mathrm{H}^{+}$ & No & Yes \\
\hline & binding/release of Lac & Yes & Yes \\
\hline & $\begin{array}{l}\text { translocation of empty } \\
\text { carrier }\end{array}$ & No & Yes \\
\hline & $\begin{array}{l}\text { translocation of loaded } \\
\text { carrier }\end{array}$ & No & Yes \\
\hline
\end{tabular}

Table 1: Predicted effect on transport for the different model configurations. 
$1 \mathrm{nM} \mathrm{H}^{+}\left(\mathrm{pH}_{0} 9.0\right)$ resulted in a fast intracellular alkalinization with a rate of $-28.1 \pm 3.1 \mathrm{nM} \mathrm{H}^{+} /$min, induced by export of lactate and $\mathrm{H}^{+}$ via the MCT1. Decreasing the extracellular $\mathrm{H}^{+}$concentration to $1 \mathrm{nM}$ and simultaneously increasing the lactate concentration to $60 \mathrm{mM}$, resulted in slow substrate efflux with a rate of $-5.0 \pm 0.3 \mathrm{nM} \mathrm{H}^{+} / \mathrm{min}$. In the same way, removal of lactate during increase of $\mathrm{H}^{+}$concentration to $1 \mu \mathrm{M}\left(\mathrm{pH}_{\mathrm{o}} 6.0\right)$ resulted in a weak intracellular alkalinization with a rate of $-3.5 \pm 0.5 \mathrm{nM} \mathrm{H}^{+} / \mathrm{min}$. It appears that the presence of a single extracellular MCT1 substrate, either protons or lactate, is sufficient to substantially decrease the rate of efflux.
According to the kinetic analysis summarized in Table 1 , the results in Figure 2 indicate two possible model configurations. The proton is either the first substrate to bind to MCT1, where the $\mathrm{H}^{+}$ binding/release is also the slowest step, or conversely, lactate is the first substrate that binds to MCT1 and lactate binding/release is the slowest step. However, since MCT1 is prone to trans-acceleration (Juel and Halestrap, 1999), the latter case could be discarded. Thus, in our description of MCT1 transport, we adopt the model consisting of the binding scheme in Figure 1 with the two proton reactions being the rate-limiting steps. Consequently, the MCT1 transport rate will be described by Eq. 14. As well as describing MCT1 transport as such,

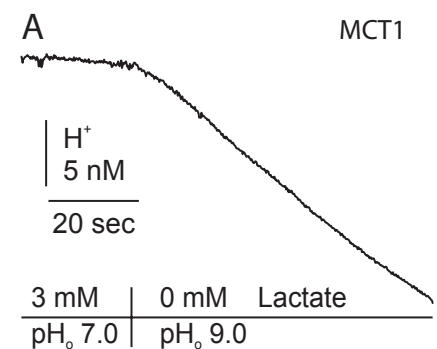

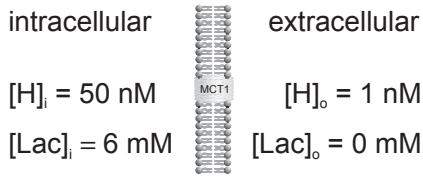

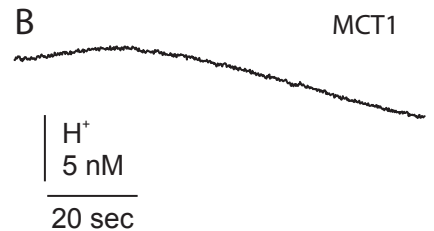

\begin{tabular}{l|ll}
$3 \mathrm{mM}$ & $60 \mathrm{mM}$ & Lactate \\
\hline $\mathrm{pH}_{\circ} 7.0$ & $\mathrm{pH}_{\circ} 9.0$
\end{tabular}

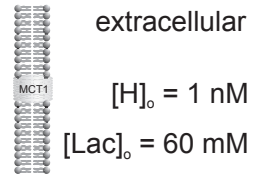

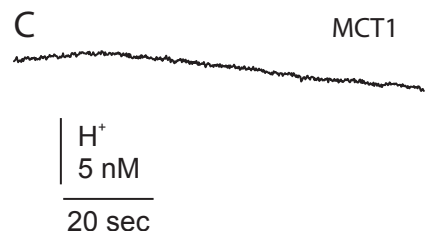

\begin{tabular}{l|l}
$3 \mathrm{mM}$ & $0 \mathrm{mM}$ Lactate \\
\hline $\mathrm{pH} 70$ & $\mathrm{pH} 6.0$
\end{tabular}

Figure 2: Proton efflux via the MCT1 as subject to extracellular $\mathrm{H}^{+}$and lactate. A MCT1-expressing oocyte was loaded with $3 \mathrm{mM}$ lactate at $\mathrm{pH}$ 7.0. Afte equilibrium was reached extracellular solution was either changed to $0 \mathrm{mM}$ lactate, $\mathrm{pH}_{\circ} 9.0(\mathbf{A}), 60 \mathrm{mM}$ lactate, $\mathrm{pH}_{\mathrm{o}} 9.0(\mathbf{B})$ or $0 \mathrm{mM}$ lactate, $\mathrm{pH}, 6.0(\mathbf{C})$. Shown is the intracellular $\mathrm{H}^{+}$concentration shortly before and during change in extracellular solution. The sketches under the original recordings show the intra- and extracellular concentration of lactate and $\mathrm{H}^{+}$at the point of change in extracellular solution.
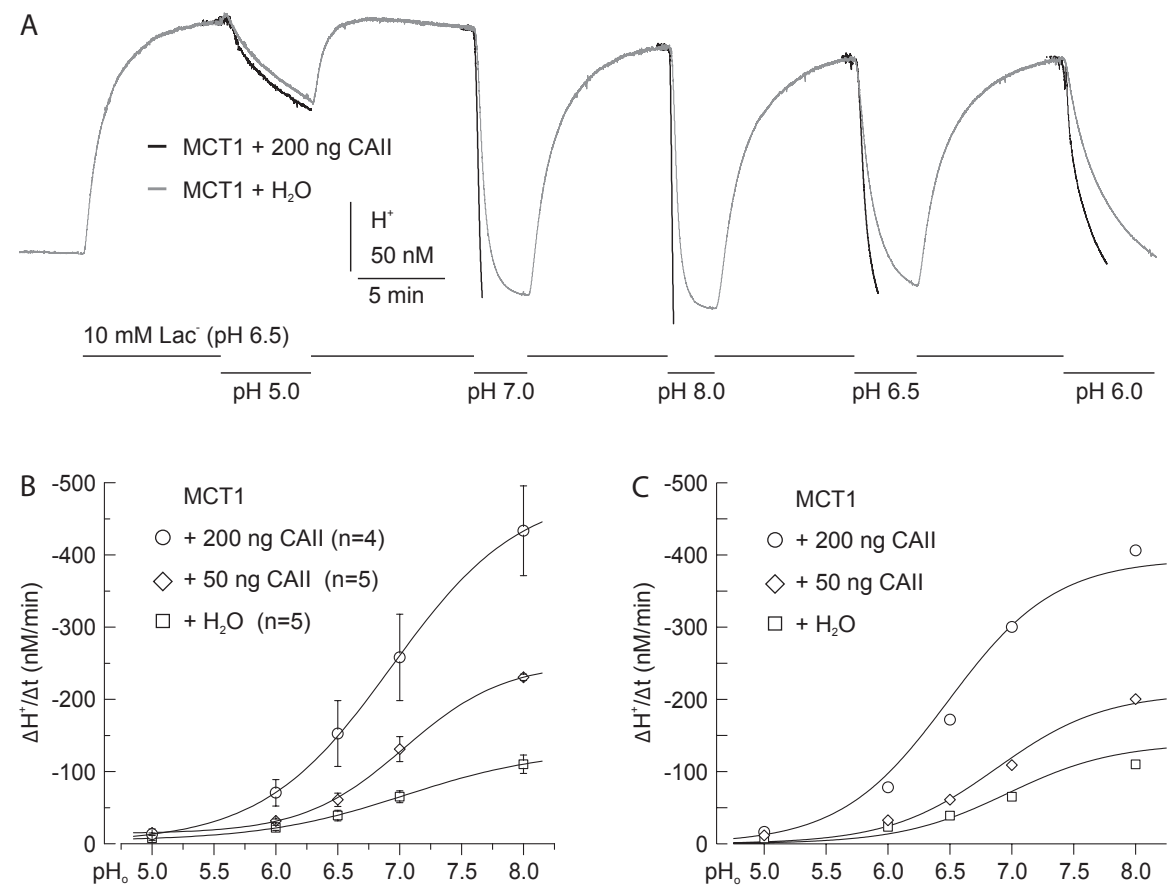

Figure 3: Dependency of $\mathrm{H}^{+}$-efflux on extracellular $\mathrm{H}^{+}$concentration. (A) Original recordings of the intracellular $\mathrm{H}^{+}$concentration in $\mathrm{MCT}$ 1-expressing oocytes injected either with $50 \mathrm{ng}$ of CAll (black trace) or $\mathrm{H}_{2} \mathrm{O}$ (gray trace), respectively, during application of $10 \mathrm{mM}$ lactate in $\mathrm{HEPES}$ buffered solution at pH ${ }_{0} 6.5$ followed by removal of lactate at extracellular $\mathrm{pH}$ of 5.0,6.0,7.0, and 8.0, respectively. (B) Rate of change in intracellular $\mathrm{H}^{+}$concentration in $\mathrm{MCT}^{-}$-expressing oocytes injected either with CAll (50 or $200 \mathrm{ng}$ ) or $\mathrm{H}_{2} \mathrm{O}$ as induced by removal of $10 \mathrm{mM}$ lactate at varying extracellular pH. (C) Simulation of the rate of change in intracellular $\mathrm{H}^{+}$ concentration in MCT1-expressing oocytes with varying concentrations of CAll as induced by removal of $10 \mathrm{mM}$ lactate at varying extracellular $\mathrm{pH}$. 

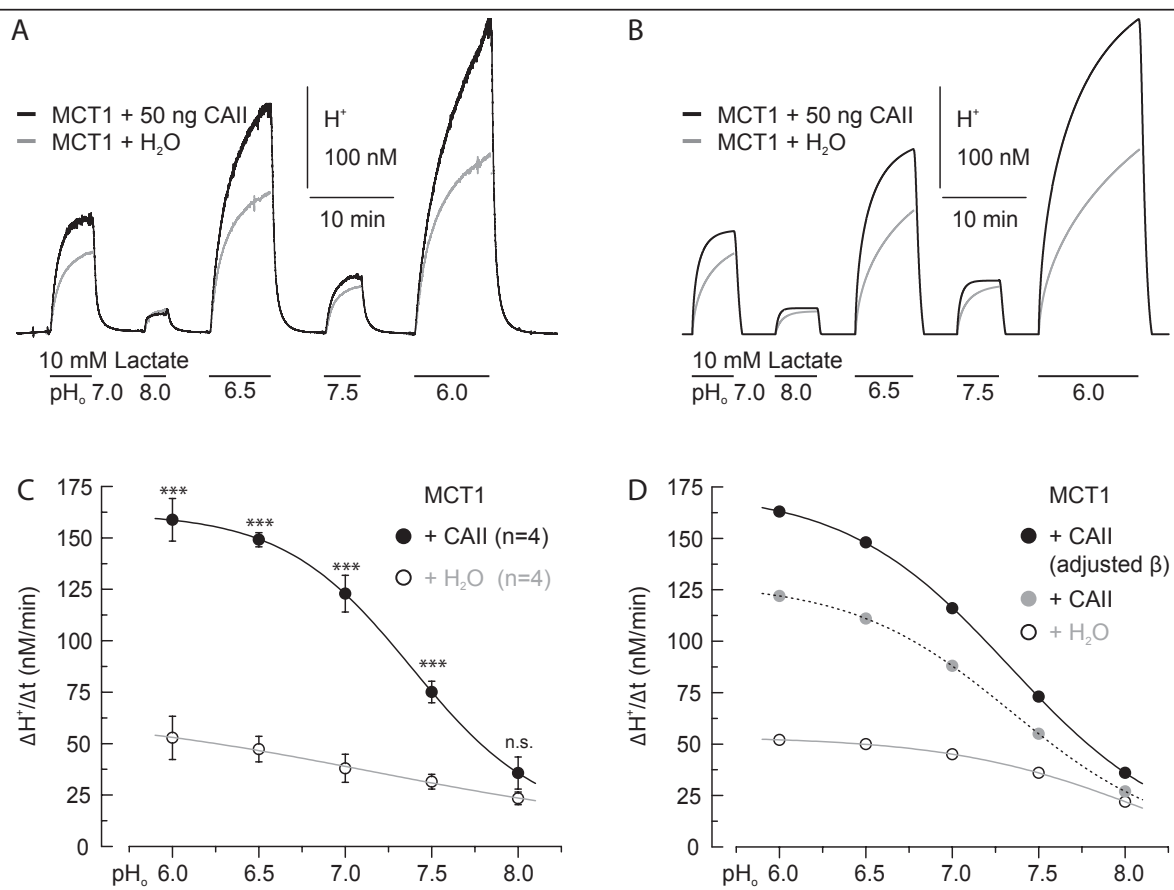

Figure 4: Dependency of $\mathrm{H}^{+}$-influx on extracellular $\mathrm{H}^{+}$concentration. (A) Original recordings of the intracellular $\mathrm{H}^{+}$concentration and $(\mathbf{B})$ simulation of the changes in intracellular $\mathrm{H}^{+}$concentration in MCT1-expressing oocytes injected either with $50 \mathrm{ng}$ of CAll (black trace) or $\mathrm{H}_{2} \mathrm{O}$ (gray trace), respectively, during application of 10 mM lactate in HEPES-buffered solution at $\mathrm{pH}$ 6.0,6.5, 7, 7.5, and 8.0, respectively. (C) Rate of rise in intracellular $\mathrm{H}^{+}$concentration in $\mathrm{MCT} 1$-expressing oocytes injected either with $50 \mathrm{ng}$ of CAll or $\mathrm{H}_{2} \mathrm{O}$ as induced by application of $10 \mathrm{mM}$ lactate at varying extracellular $\mathrm{pH}$. (D) Simulation of the rate of rise in intracellular $\mathrm{H}^{+}$concentration in MCT1-expressing oocytes with and without CAll as induced by application of $10 \mathrm{mM}$ lactate at varying extracellular $\mathrm{pH}$. Simulation of CAll-injected cells is performed at two different levels of intracellular buffer capacity.

Eq. 14 is the starting point for modeling the interaction between MCT1 and CAII.

CAII is believed to enhance transport activity of MCT1 either by removing $\mathrm{H}^{+}$from the transporter pore or supplying the transporter with $\mathrm{H}^{+}$via a proton shuttle along a chain of CAII at the cells inner membrane surface and an enhanced exchange of $\mathrm{H}^{+}$with the cytosol (Becker \& Deitmer, unpublished), taking direct influence on the rate of proton binding and release at the MCT1. Since the binding and release of the proton are the only reactions in the model that are not constantly in equilibrium, a change in the corresponding rate constants could change the transport rate without altering the equilibrium. Additionally, it has to be taken into consideration that CAII is only present intracellularly, which makes any effect on $k_{56}$ and $k_{65}$ unlikely. As a consequence, interaction between MCT1 and CAII will be modeled as a more efficient way of proton exchange between the cytosol bulk and the MCT1 proton target site. Both $k_{12}$ and $k_{21}$ are increased with a factor $g_{C A}$, representing the effect of CAII. In the case with no CAII, $g_{C A}$ was set to one. The model reduction procedure was repeated with Eq. 12 replaced with

$$
g_{C A} k_{12}\left[H_{i n}^{+}\right] M_{1}-g_{C A} k_{12} M_{2}=k_{56} M_{5}-k_{65}\left[H_{e X}^{+}\right] M_{6}
$$

which leads to a new rate expression including the impact of CAII in which the parameters must still obey Eq. 20.

$T_{C A}=g_{C A} M_{\text {tot }} \frac{k_{12} k_{56} K_{i} K_{l}\left[H_{i n}^{+}\right]\left[L a c_{i n}^{-}\right] k_{21} k_{65} K_{o} K_{e}\left[H_{e x}^{+}\right]\left[L a c_{e x}^{-}\right]}{A^{\prime}\left[H_{i n}^{+}\right]+B\left[H_{e x}^{+}\right]+C\left[L a c_{i n}^{-}\right]+D^{\prime}\left[L a c_{e x}^{-}\right]}$

where $B$ and $C$ are the same as before, but with $A$ and $D$ replaced with $A^{\prime}$ and $D^{\prime}$ now including the $g_{C A}$ factor
$A^{\prime}=g_{C A} K_{12}\left(K_{i} K_{l}\left[L a c_{i n}^{-}\right]+K_{o}\left[\operatorname{Lac}_{e X}^{-}\right]+K_{i} K_{o}\left(K_{l}+1\right)\left[L a c_{i n}^{-}\right]\left[L a c_{e X}^{-}\right]\right)$

$$
D^{\prime}=g_{C A} k_{21} \mathrm{~K}_{o}\left(\mathrm{~K}_{e}+1\right)
$$

Hence, $g_{C A}$ now appears both in the numerator and in some of the terms in the denominator. Thus, the effect of making $g_{C A}$ larger than one will depend on the proportions of the denominator terms. If the terms containing $g_{C A}$ are dominating, the effect of increasing $g_{C A}$ will be small. On the other hand, if the terms not containing $g_{C A}$ are dominating, increasing $g_{C A}$ will lead to a significant increase in the total transport rate.

The rate expression in Eq. 22 can now be compared to the data obtained from an efflux experiment with no lactate at the outside, referred to as zero-trans efflux experiment. MCT1-expressing oocytes, either injected with 50 or $200 \mathrm{ng}$ of CAII, or an equivalent volume of $\mathrm{H}_{2} \mathrm{O}$ were loaded with $10 \mathrm{mM}$ lactate at a $\mathrm{pH}_{\circ}$ of 6.5 . After equilibrium was reached, lactate was removed and $\mathrm{pH}_{0}$ was changed to a value between 5.0 and 8.0. Figure 3A shows the original recordings of the intracellular $\mathrm{H}^{+}$concentration of MCT1-expressing oocytes either injected with $200 \mathrm{ng}$ of CAII (black trace) or $\mathrm{H}_{2} \mathrm{O}$ (grey trace). The values of the measurements are given in Figure 3B. Rate of intracellular $\mathrm{H}^{+}$concentration changes during removal of lactate are plotted against the extracellular $\mathrm{pH}$ value. Injection of CAII leads to an increase in the rate of change in intracellular $\mathrm{H}^{+}$concentration $\left(\Delta \mathrm{H}^{+} / \Delta \mathrm{t}\right)$. The magnitude of the increase grows for higher extracellular $\mathrm{pH}$, but the relative increase stays at a similar level throughout the whole $\mathrm{pH}$-range. The increase in transport activity was further amplified by an increased concentration of injected CAII. Modeling of the zero-trans efflux experiment offers the advantage that substrate 

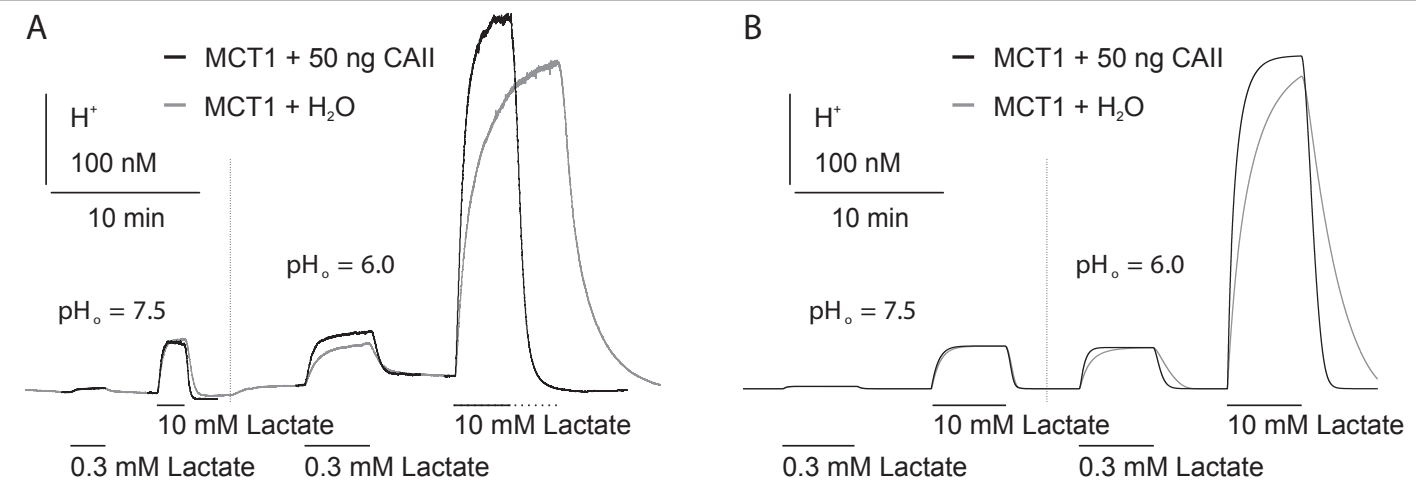

Figure 5: Dependency of MCT1 transport activity on substrate concentration. (A) Original recordings of the intracellular $\mathrm{H}^{+}$concentration and $(\mathbf{B})$ simulation of the changes in intracellular $\mathrm{H}^{+}$concentration in MCT1-expressing oocytes injected with $50 \mathrm{ng}$ of CAll (black trace) or $\mathrm{H}_{2} \mathrm{O}$ (gray trace), respectively, during application of 0.3 and $10 \mathrm{mM}$ lactate in HEPES buffered solution at $\mathrm{pH}_{\circ} 7.5$ and 6.0 .

concentrations on the trans-side are kept constant, because the bath solution is continuously replaced, which prevents any accumulation of lactate on the trans-side. Putting $\left[\mathrm{Lac}_{e x}^{-}\right]$to zero, Eq. 22 reduces to

$$
T_{C A}=g_{C A} M_{t o t} \frac{k_{12} k_{56}\left[H_{i n}^{+}\right]}{g_{C A} k_{12}\left[H_{i n}^{+}\right]+k_{65} K_{e}\left[H_{e x}^{+}\right]+k_{56}\left(K_{e}+1\right)}
$$

Interestingly, this rate is independent of the intracellular lactate concentration (as long as this concentration is not zero since that would yield a zero rate for obvious reasons). To obtain the initial rate of efflux following a change in the bath solution, it is sufficient to insert the known values of intra and extracellular $\mathrm{pH}$. Figure $3 \mathrm{C}$ shows the calculated $\mathrm{pH}_{0}$ dependence of Eq. 25 together with the data points from Figure 3B. The parameters $k_{12}$ and $k_{65}$ are assumed to be $4 \times 10^{7} \mathrm{mM}^{-1} \mathrm{~s}^{-1}$, corresponding to diffusion-limited protonation (Ädelroth and Brzezinski, 2004). A best fit to data was found for $k_{56}$ $=2.6 \times 10^{3} \mathrm{~s}^{-1}, K_{e}=4.5$, and $g_{C A}=1,2$, and 5 for 0,50 , and $200 \mathrm{ng}$ of CAII, respectively.

To further investigate the effect of extracellular $\mathrm{pH}$ on the CAIIinduced increase in MCT1 transport activity, we measured the change in intracellular $\mathrm{H}^{+}$concentration in MCT1-expressing oocytes, either injected with $50 \mathrm{ng}$ of CAII or an equivalent amount of $\mathrm{H}_{2} \mathrm{O}$, during application of $10 \mathrm{mM}$ lactate at different extracellular $\mathrm{pH}$ values between 6.0 and 8.0 (Figure 4A). Transport activity of MCT1 was determined by measuring $\Delta \mathrm{H}+/ \Delta \mathrm{t}$. Both for CAIl-injected as well as for $\mathrm{H}_{2} \mathrm{O}$-injected oocytes, a decrease in extracellular $\mathrm{pH}$ resulted in an increase in MCT1 transport activity. Injection of CAII augmented $\mathrm{pH}$ dependency of MCT1 transport activity, increasing $\Delta H+/ \Delta t$ to a larger extent at low extracellular $\mathrm{pH}$ values, while at high $\mathrm{pH}$, only a small CAII-induced increase in MCT1 activity could be observed (Figure 4C). The injection of CAII lead to a more sigmoid shaped curve, compared to the curve observed in MCT1-expressing oocytes without CAII. Figure $4 \mathrm{~B}$ shows the simulation of the experiment shown in Figure 4A. The initial flux rates from the simulation are shown in Figure 4D. Even though the experiment starts from zero- trans conditions, substrates will continuously accumulate in the cell. For large fluxes, accumulation is so rapid that even during the $20 \mathrm{~s}$ time interval where the lactate-induced acidification shows nearly linear kinetics, which is used to measure $\Delta H+/ \Delta t$, a counter-flow may have developed. This motivates the use of Eq. 22 in the simulations. The parameter values used to fit the data were $K_{i}=K_{o}=0.5 \mathrm{mM}^{-1}, K_{\mathrm{e}}=K_{l}=4.5, k_{56}=k_{21}$ $=1 \times 10^{3} \mathrm{~s}^{-1}$, and $g_{C A}=4$, showing a somewhat lower value of $k_{56}$ and a higher value of $g_{C A}$ compared to the simulations in Figure 3.

The simulated rate of substrate influx for CAII-injected cells comes in two variants. One where the intracellular buffer capacity is decreased compared to cells without CAII (black trace), and one where the buffer capacity is left unchanged at the level of $\mathrm{H}_{2} \mathrm{O}$ cells (dotted curve). The former fits the data of the measurements shown in Figure $4 \mathrm{C}$ better, but the latter illustrates the properties of the model more clearly. Nevertheless, in both cases CAII-induced enhancement of inward transport has a strong dependence on extracellular pH. For higher $\mathrm{pH}_{\mathrm{o}}$ the percental increase in transport rate is smaller than for lower $\mathrm{pH}_{0}$. As the dotted and grey traces only differ by $g_{C A}$, this emphasizes the effect of CAII unclouded by putative differences in oocyte batches. We have previously observed that there is nearly no CAII-induced increase in MCT1-expressing oocytes at $\mathrm{pH}_{0} 7.5$, while at $\mathrm{pH}_{\mathrm{o}} 6.0$ a strong effect occurred (Becker \& Deitmer, unpublished).

Finally, the effect of different extracellular concentrations of lactate was investigated by influx experiments. Figure 5A shows the original recordings of intracellular $\mathrm{H}^{+}$concentration of two MCT1expressing oocytes, either injected with $50 \mathrm{ng}$ of CAII (black trace) or $\mathrm{H}_{2} \mathrm{O}$ (grey trace) during application of lactate at very low and at high concentration $(0.3 \mathrm{mM}$ and $10 \mathrm{mM})$, at low and high extracellular $\mathrm{pH}$ $\left(\mathrm{pH}_{\mathrm{o}} 6.0\right.$ and $\mathrm{pH}_{\mathrm{o}}$ 7.5). The measurements indicate that the increase in transport activity induced by CAII is most evident at $\mathrm{pH}_{\mathrm{o}} 6.0$, and only marginal at $\mathrm{pH}_{0}$ 7.5. The concentration of lactate does not seem to affect the CAII-dependent enhancement of MCT1 transport. Intracellular resting $\mathrm{pH}$ of MCT1-expressing oocytes injected with CAII or $\mathrm{H}_{2} \mathrm{O}$ was $7.38 \pm 0.03$ and $7.33 \pm 0.02$, respectively. Figure $5 \mathrm{~B}$ shows the simulation of the experiment as described in Figure $5 \mathrm{~A}$ with the same parameters as in Figure 4.

\section{Discussion}

In the present study we investigated transport function of the MCT1 and its interaction with the enzyme CAII by means of electrophysiological measurements of MCT1 activity in Xenopus oocytes and by mathematical modeling of the transport process. Viewing the transport process as a series of sub-steps, assumptions about which step is the rate-limiting one will play a crucial role in the resulting kinetics. Based on single substrate inhibition experiments we propose, that association, and dissociation, of the proton is the step that limits the turnover-rate of the symport. These findings are in contrast to earlier studies stating that the translocation of the empty carrier is the rate-limiting step (Halestrap and Meredith, 2004; Deuticke, 1982). However, combining the assumption of $\mathrm{H}^{+}$ binding and release being the rate-limiting step with the previously suggested ordered binding scheme, where the proton binds, and is released, first (de Bruijne et al., 1983), is still compatible with the 
fast exchange experiments carried out earlier (Deuticke, 1982) and the single substrate inhibition, but opens up a natural role for CAII as an accelerator of proton reactions. When testing the impact of different binding order schemes on the reduced rate expression, some configurations that occasionally appear in similar studies were omitted. Glide symmetry, the opposite of mirror symmetry, means that the substrate that binds first is also released first. Since the conclusion that the proton binding/release is the slowest step does not depend on the intracellular binding order, glide symmetry could not be ruled out by the single substrate inhibition experiments used in this study. However, any model where substrate binding/ release is rate-limiting means that glide symmetry must be ruled out on the basis of the accelerated exchanges experiments (Deuticke, 1982). Another binding mechanism often considered is the so called random binding (Lytle et al., 1998; Hopfer and Groseclose, 1980). Here, either substrate may bind or dissociate first, determined by some probabilistic mechanism. Based on the measurements in this study, random binding cannot be excluded as a possible mechanism. Nevertheless, this is not surprising, since all ordered binding schemes are just special cases of the more general random binding.

The main results of this study are the binding order scheme in Figure 1 and the rate expression in Eq. 22. The implications of these results can be summarized as follows. During net transport of protons and lactate via the MCT1, the proteins must pass through two bottleneck reactions, the binding and release of protons on both sides of the membrane. Depending on the substrate concentrations and the parameters of the model, one of these bottlenecks may be dominating. For the special cases of zero-trans influx or efflux (setting $\left[\mathrm{Lac}_{i n}^{-}\right]$or $\left[\mathrm{Lac}_{\mathrm{ex}}^{-}\right]$to zero in Eq. 22), this will be determined by the $\mathrm{pH}$ at both sides of the membrane, the rate constants for the proton binding/release, and any bias towards intra or extracellular location of the empty carrier. If the bottleneck should be between states $M_{1}$ and $M_{2}$, the presence of CAIl will have an enhancing effect on transport. In the efflux experiments, CAII has a similar sized effect over the whole range of $\mathrm{pH}_{0}$. Here, the intracellular binding and release of protons limits the process irrespectively of $\mathrm{pH}_{\mathrm{o}}$, a consequence of the asymmetric distribution of empty carriers $(K$ $=4.5$ ). For the influx of substrate via the MCT1 on the other hand, the effect of CAII decreases quickly as $\mathrm{pH}_{\mathrm{o}}$ increases. For high values of $\mathrm{pH}_{\mathrm{o}}$, the extracellular binding and release of protons becomes the dominating bottleneck and the CAII reinforcement on the corresponding intracellular reactions makes little or no difference. An interesting property of Eq. 22 can be seen by putting either $\left[\mathrm{Lac}_{i n}^{-}\right]$or $\left[\mathrm{Lac}_{e x}^{-}\right]$to zero. In both cases the dependence of the remaining lactate concentration vanishes. According to the model, the dependence of influx rate on lactate concentration is therefore only a consequence of a developing counter-transport.

In Eq. 21, the effect of CAII was included in the model as an increase of $k_{12}$ and $k_{21}$ with the common factor $g_{C A}$. This was motivated by assuming that proton exchange with the cytosol bulk was made more effcient by CAII. We will now elaborate on this idea. To start with, an observation from Figure 5 gives an important hint of how the CAII mechanism works. By adjusting the lactate concentration, fluxes of similar magnitude can be obtained for different extracellular $\mathrm{pH}$. The flux rate at $10 \mathrm{mM}$ lactate, $\mathrm{pH}_{0} 7.5$, is of the same size as the flux rate at $0.3 \mathrm{mM}, \mathrm{pH}_{\mathrm{o}} 6.0$. However, the effect of CAII appears only to depend on the extracellular $\mathrm{pH}$, not on the lactate concentration or the magnitude of the flux. The distribution of MCT1 proteins over the six states $M_{1}-M_{6}$ is the only way of accessing information about extracellular substrate concentrations at the inner side of the membrane. Therefore, two extracellular conditions yielding the same flux cannot be distinguished by any hypothetical CAII mechanism that is not linked to the reactions included in the kinetic model. In terms of the model, MCT1-CAII interaction is then restricted to alterations in the parameters of Eq. 22. Actually, since there is an evident effect of CAII in the efflux experiments (where $\left[\mathrm{Lac}_{e x}^{-}\right]$is zero), parameter alteration must include $k_{12}, k_{56}, k_{65}$ or $K_{e .}$ Changes in $k_{56}$ and $k_{65}$ are not very intuitive because of the intracellular location of CAII, but cannot be dismissed with absolute certainty. Moreover, an additional constraint on the parametric effect of CAII is Eq. 20, which always has to be satisfied due to thermodynamic considerations. Decreasing $K_{e}$, coupled with an appropriate change in (at least) one other parameter, could enhance zero-trans efflux, but the fit to the data obtained in the measurements is worse and for the influx experiment such changes give clearly erroneous results. These findings point to a change in $k_{12}$ as the most likely effect of CAII on MCT1 within the model. Again, at least one more parameter must also be altered for Eq. 20 to hold. An equally sized increase in $k_{21}$ is the only coupled alteration that gives the desired effect also for the influx experiments. A mutual increase of $k_{12}$ and $k_{21}$ with the factor $g_{C A}$ preserves the equilibrium of the reaction between $M_{1}$ and $M_{2}$, and gives CAII an enhancing effect on transport activity, without shifting the equilibrium. The CAII-induced enhancement of transport is not dependent on the enzymes catalytic activity (Becker et al., 2005), speeding up the interconversion of $\mathrm{CO}_{2}$ and $\mathrm{HCO}_{3}^{-}$, suggesting that the enhancement is not due to a faster equilibration with the $\mathrm{CO}_{2}$ buffer system. Thus, the increase of the intracellular rate constants for binding and release of protons in the model must have a different physical interpretation. We hypothesize that by binding to MCT1, CAII provides additional binding sites for protons. Protonatable residues that are up to $12 \AA$ apart from each other, could form proton-attractive domains, and could share the proton among them at a very fast rate, exceeding the upper limit of diffusion-controlled reactions (Gutman et al., 2006). Negatively charged residues of membrane proteins with overlapping Coulomb cages can form a "proton-collecting antenna" that collects protons from solution and "funnels" them to the entrance of a proton-transfer pathway, or vice versa, can remove $\mathrm{H}^{+}$from the side of a transporter and pass them to the bulk solution (Bränden et al., 2006; Georgievskii et al., 2002). By a collectively operating network of proton binding sites, established by CAII, the protonation and deprotonation rate of the MCT1 proton site could be accelerated, thereby increasing the overall rate of transport.

A question about the CAll-enhanced flux rate that needs to be addressed given the proposed antenna effect, is whether it relies on binding of a single CAII molecule to an MCT1 or on some other principle. In the first scenario, CAII moves around in the vicinity of the membrane surface, reversibly binding to MCT1. The fraction of MCT1CAII complexes would then be dependent on the concentrations of both MCT1 and CAII. A more realistic model, instead of the model described by Eq. 22, would be a linear combination of Eq. 15 and Eq. 22, $f T_{C A}+(1-f) T$, where $f \in[0,1]$ is the fraction of CAII-bound transporters. However, this model works worse due to the following reason: Comparing the effect of 50 and $200 \mathrm{ng}$ CAII on transport activity during efflux of lactate reveals that the enhancing effect is half-saturated at $\sim 200 \mathrm{ng}$. This would mean that approximately $15 \%$ of the MCT1 are bound to a CAII at $50 \mathrm{ng}$. Since the other $85 \%$ would operate in the non-enhancing mode, the small fraction of MCT1CAII would have to cope with the overall transport enhancement on their own. Such a high enhancement of only a small fraction of the MCT1 proteins is not compatible with Eq. 22, as it saturates for high 
values of $g_{C A}$ before reaching the amplification needed to explain the observed transport rates. Another imaginable scenario is that the combination reaction of the two proteins is saturated already at an injection of $50 \mathrm{ng}$ of CAII, resulting in a protein population more or less made up of MCT1-CAII complexes alone. A suggestion for how the enhancement mechanism still could be dependent on the CAII concentration would be that the CAII molecules themselves can bind, forming clusters or chains. Higher concentrations of CAII would mean larger CAII- structures connected to each MCT1, which in turn also could mean more effective antennas. In this scenario each MCT1 contributes a little to the overall enhancement, something that works better in the model compared to the two-by-two case where a few MCT1 give a large contribution.

A simple model of the intracellular environment makes analysis of the MCT1 transport easier. Because of this, we have chosen to describe the concentrations of substrates and buffers as spatially homogenous although we know that this is an approximation. We use an "effective volume", smaller then the real cell volume, as an approximation of what happens in the vicinity of the electrode. For a more exact and realistic description of the intracellular species it would be necessary to employ a diffusion-reaction system governed by partial differential equations. Without a more detailed model it is hard to draw precise conclusions about exact parameter values. However, the homogenous approximation is a simplification that seems sufficient to explain the basic principles of MCT1 transport, and its enhancement by CAII.

\section{Abbreviations and Model Notation \\ CAll carbonic anhydrase isoform II \\ $g_{C A} \quad$ model parameter describing the effect of CAll \\ $\mathrm{H}^{+}{ }_{\mathrm{CA}} \quad$ intracellular proton concentration \\ $\mathrm{H}_{\mathrm{ex}}^{+}$model variable describing the extracellular proton concentration \\ $\left[H_{i n}^{+}\right]$model variable describing the intracellular proton concentration \\ $\left[\mathrm{Lac}_{\mathrm{c}}^{-}\right]$model variable describing the extracellular lactate concentration \\ [ Lacii $\left._{\text {iin }}^{-}\right]$model variable describing the intracellular lactate concentration \\ $k_{x y} \quad$ model rate constant \\ $K_{x y} \quad$ model equilibrium constant \\ $\mathrm{K}_{m} \quad$ Michaelis constant \\ $M \quad$ model variable describing transporter state \\ $M^{\mathrm{s}} \quad$ distribution of transporter states \\ $M_{\text {tot }} \quad$ model parameter describing the total number of transporters \\ MCT monocarboxylate transporter \\ ODE ordinary differential equation \\ $\mathrm{pH} \quad$ intracellular $\mathrm{pH}$ \\ $\mathrm{pH}^{-} \quad$ extracellular $\mathrm{pH}$ \\ $\mathrm{pK}_{\mathrm{a}}^{\circ} \quad$ logarithmic measure of the acid dissociation constant \\ $T$ model variable describing the rate of MCT1 transport \\ $T_{C A} \quad$ model variable describing the rate of MCT1 transport in presence of CAll \\ $\mathrm{V}_{\max } \quad$ maximum reaction rate \\ Acknowledgements}

This research is supported by the Centre of Mathematical and Computational Modelling at the University of Kaiserslautern, the Deutsche Forschungsgemeinschaft (De 231/16-4), the Swedish Foundation for Strategic Research and the Gothenburg Mathematical Modelling Centre, which are all gratefully acknowledged.

\section{References}

1. Adelroth PA, Brzezinski P (2004) Surface-mediated proton-transfer reactions in membrane-bound proteins. Biochim Biophys Acta 1655:102-115.

2. Bangsbo J, Juel C, Hellsten Y, Saltin B (1997) Dissociation between lactate and proton exchange in muscle during intense exercise in man. J Physiol 504 : 489-499.

3. Becker HM, Deitmer JW (2007) Carbonic anhydrase II increases the activity of the human electrogenic $\mathrm{Na}^{+} / \mathrm{HCO}_{3}^{-}$cotransporter. J Biol Chem 282: 1350813521

4. Becker HM, Bröer S, Deitmer JW (2004) Facilitated lactate transport by
MCT1 when coexpressed with the sodium bicarbonate cotransporter (NBC) in Xenopus oocytes. Biophys J 86: 235-247.

5. Becker HM, Fecher-Trost C, Hirnet D, Sültemeyer D, Deitmer JW (2005) Transport activity of MCT1 expressed in Xenopus oocytes is increased by interaction with carbonic anhydrase. J Biol Chem 280: 39882-39889.

6. Bergersen LH (2007) Is lactate food for neurons? Comparison of monocarboxylate transporter subtypes in brain and muscle. Neuroscience 145 $11-19$

7. Bouzier-Sore AK, Serres S, Canioni P, Merle M (2003) Lactate involvement in neuron-glia metabolic interaction: (13)C-NMR spectroscopy contribution Biochimie 85: 841-848.

8. Bower JM, Bolouri H (2001) Computational modeling of genetic and biochemical networks. MIT Press.

9. Bränden M, Sanden T, Brzezinski P, Widengren J (2006) Localized proton microcircuits at the biological membranewater interface. PNAS 103: 1976619770.

10. Bröer S, Rahman B, Pellegri G, Pellerin L, Martin JL, et al. (1997) Comparison of lactate transport in astroglial cells and monocarboxylate transporter 1 (MCT1) expressing xenopus laevis oocytes. expression of two different monocarboxylate transporters in astroglial cells and neurons. J Biol Chem 272 : 30096-30102.

11. Bröer S, Schneider HP, Bröer A, Rahman B, Hamprecht B, et al. (1998) Characterization of the monocarboxylate transporter 1 expressed in Xenopus laevis oocytes by changes in cytosolic pH. Biochem J 333: 167-174.

12. Brooks GA (1998) Mammalian fuel utilization during sustained exercise. Comp Biochem Physiol B Biochem Mol Biol 120: 89-107.

13. de Bruijne AW, Vreeburg H, van Stevenick J (1983) Kinetic analysis of L-lactate transport in human erythrocytes via the monocarboxylate-specific carrie system. Biochim Biophys Acta 732: 562-568

14. Debernardi R, Pierre K, Lengacher S, Magistretti PJ, Pellerin L (2003) Cellspecific expression pattern of monocarboxylate transporters in astrocytes and neurons observed in different mouse brain cortical cell cultures. J Neurosci Res 73: 141-155.

15. Deitmer JW (1991) Electrogenic sodium-dependent bicarbonate secretion by glial cells of the leech central nervous system. J Gen Physiol 98: 637-655.

16. Deitmer J W, Rose CR (1996) pH regulation and proton signalling by glial cells Prog Neurobiol 48: 73-103

17. Deuticke B (1982) Monocarboxylate transport in erythrocytes. J Membrane Biol 70:89-103

18. Fall CP, Marland ES, Wagner JM, Tyson JJ (2002) Computational cell biology. Springer-Verlag

19. Galic S, Schneider HP, Bröer A, Deitmer JW, Bröer S (2003) The loop between helix 4 and helix 5 in the monocarboxylate transporter MCT1 is important for substrate selection and protein stability. Biochem J 376: 413-422.

20. Georgievskii Y, Medvedev ES, Stuchebrukhov AA (2002) Proton transport via the membrane surface. Biophys J 82: 2833-2846.

21. Gutman M, Nachliel E, Friedman R (2006) The dynamics of proton transfer between adjacent sites. Photochem Photobiol Sci 5:531-537.

22. Halestrap AP, Meredith D (2004) The SLC16 gene family - from monocarboxylate transporters (MCTs) to aromatic amino transporters and beyond. Pflugers Arch 447: 619-628.

23. Hashimoto T, Masuda S, Taguchi S, Brooks GA (2005) Immunohistochemica analysis of MCT1, MCT2 and MCT4 expression in rat plantaris muscle. $J$ Physiol 567: 121-129.

24. Hopfer T, Groseclose R (1980) The mechanism of $\mathrm{Na}^{+}$-dependent D-Glucose transport. J Biol Chem 255: 4453-4462.

25. Juel C (2001) Current aspects of lactate exchange: lactate $/ \mathrm{H}^{+}$transport in human skeletal muscle. Eur J Appl Physiol 86: 12-16.

26. Juel C and Halestrap AP (1999) Lactate transport in skeletal muscle - role and regulation of the monocar-boxylate transporter. J Physiol 517: 633-642.

27. Juel C, Grunnet L, Holse M, Kenworthy M, Sommer V, et al. (2001) Reversibility 
Citation: Almquist J, Lang P, Prätzel-Wolters D, Deitmer JW, Jirstrand M, et al. (2010) A Kinetic Model of the Monocarboxylate Transporter MCT1 and its Interaction with Carbonic Anhydrase II. J Comput Sci Syst Biol 3: 107-116. doi:10.4172/jcsb.1000066

of exercise-induced translocation of $\mathrm{Na}^{+}-\mathrm{K}^{+}$pump subunits to the plasma membrane in rat skeletal muscle. Pflugers Arch 443: 212-217.

28. Juel C, Lundby C, Sander M, Calbet JAL, van Hall G (2003) Human skeletal muscle and erythrocyte proteins involved in acid-base homeostasis: adaptations to chronic hypoxia. J Physiol 548:639-648.

29. Klipp E, Herwig R, Kowald A, Wierling C, Lehrach H (2005) Systems biology in practice. Wiley-VCH.

30. Li X, Alvarez B, Casey JR, Reithmeier RA, Fliegel L (2002) Carbonic anhydrase II binds to and enhances activity of the $\mathrm{Na}^{+} / \mathrm{H}^{+}$exchanger. J Biol Chem 277: 36085-36091.

31. Lytle C, McManus TJ, Haas M (1998) A model of Na-K-2Cl cotransport based on ordered ion binding and glide symmetry. Am J Physiol Cell Physiol 274 299-309

32. Magistretti PJ, Pellerin L, Rothman DL, Shulman RG (1999) Energy on demand. Science 283: 496-497.

33. Pellerin L, Pellegri G, Bittar PG, Charnay Y, Bouras C, et al. (1998) Evidence supporting the existence of an activity-dependent astrocyte-neuron lactate shuttle. Dev Neurosci 20: 291-299.

34. Poole RC, Sansom CE, Halestrap AP (1996) Studies of the membrane topology of the rat erythrocyte $\mathrm{H}^{+} /$lactate cotransporter (MCT1). Biochem J 320: 817-824.

35. Pushkin A, Abuladze N, Gross E, Newman D, Tatishchev S, et al. (2004) Molecular mechanism of kNBC1-carbonic anhydrase II interaction in proximal tubule cells. J Physiol 559:55-65.
36. Schmidt H (2005) SBTOOLBOX: Systems Biology Toolbox for MATLAB.

37. Schmidt H (2006) SBADDON: High performance simulation interface and parameter estimation framework for MATLAB.

38. Schurr A, West CA, Rigor BM (1988) Lactate-supported synaptic function in the rat hippocampal slice preparation. Science 240: 1326-1328.

39. Svichar N, Chesler M (2003) Surface carbonic anhydrase activity on astrocytes and neurons facilitates lactate transport. Glia 41: 415-419.

40. Szallasi Z, Stelling J, Periwal V (2006) System modeling in cellular biology MIT Press.

41. Thomas C, Bishop D, Moore-Morris T, Mercier J (2007) Effects of high-intensity training on MCT1, MCT4, and NBC expressions in rat skeletal muscles: influence of chronic metabolic alkalosis. Am J Physiol Endocrinol Metab 293: E916-22.

42. Vince JW, Reithmeier RA (1998) Carbonic anhydrase II binds to the carboxy terminus of human band 3 , the erythrocyte $\mathrm{Cl}^{+} / \mathrm{HCO}_{3}^{-}$exchanger. J Biol Chem 273: $28430-28437$.

43. Wetzel P, Hasse A, Papadopoulos S, Voipio J, Kaila K, et al. (2001) Extracellula carbonic anhydrase activity facilitates lactic acid transport in rat skeletal muscle fibres. J Physiol 531:743-756.

44. Wilson MC, Jackson VN, Heddle C, Price NT, Pilegaard H, et al. (1998) Lactic acid efflux from white skeletal muscle is catalyzed by the monocarboxylate transporter isoform MCT3. J Biol Chem 273: 15920-15926. 